\title{
Notas sobre las repercusiones del affaire Caillaux en la prensa de Buenos Aires
}

\author{
Emiliano Gastón Sánchez
}

Universidad Nacional de Tres de Febrero / CONICET

L'affaire Caillaux es una novela apasionante, tanto en Francia como en Bélgica misma; desde l'affaire Dreyfus no ha habido otro de igual o parecido interés.

Roberto J. Payró ${ }^{1}$

$\mathrm{L}$ a tarde del 16 de marzo de 1914, Henriette Raynouard, esposa en segundas nupcias de Joseph Caillaux -ministro de Finanzas del gobierno francés y líder del Partido Radical- ingresó en la redacción del diario Le Figaro, ubicada en el 26 rue Drouot de la capital francesa, y asesinó a balazos a su director, Gaston Calmette. ${ }^{2}$ Desde hacía varios meses, el ministro era objeto de una feroz campaña en su contra vehiculizada por el matutino, la cual adquirió su punto más alto con la publicación de algunas cartas intercambiadas con su primera esposa, Berthe Gueydan. El 13 de marzo la portada del diario exhibió la

\footnotetext{
1 "Cartas informativas. Amenazas (Para La Nación). Bruselas, julio de 1914", La Nación, 19 de agosto de 1914 , p. 4, destacado en el original. Se han conservado la ortografía y la puntuación originales en las citas de las fuentes.

2 Para una caracterización más detallada del hecho, véase Edward Berenson, The Trial of Madame Caillaux, California, University of California Press, 1992, pp. 1-3; y Jean-Yves Le Naour, Meurtre au Figaro. L'affaire Caillaux, París, Larousse, 2007, pp. 11-15.
}

reproducción de una carta de puño y letra de Caillaux en la que brindaba detalles de su trabajo en la Cámara en relación con un proyecto de impuesto a la renta. La publicación de la misiva tenía por objeto mostrar al ministro como un ejemplo de doble moral; no obstante, hubo un pequeño detalle que no pasó inadvertido y motivó los comentarios jocosos de la opinión francesa: el modo familiar y cariñoso con el que Caillaux se dirigía hacia su primera esposa, "Ton Jo". ${ }^{3}$ Pocos días después, en su edición del 16 de marzo, el matutino anunció que continuaría con la publicación de su correspondencia privada, ${ }^{4}$ lo que provocó la angustia y la furia de la mujer que vació el cargador de su pistola Browning sobre la humanidad del periodista. Se iniciaba así el llamado affaire Caillaux, también conocido como "el crimen de Le Figaro", un escándalo político y mediático que mantuvo en vilo a la prensa y a la opinión pública de Francia (y presumiblemente de buena parte del

\footnotetext{
3 "La véritable déclaration de $\mathrm{M}$. Caillaux relative à l'impôt sur le revenu" y "La preuve des machinations secrètes de M. Caillaux", Le Figaro, 13 de marzo de 1914 , p. 1.

4 "Intermède comique. Les notes biographiques de 'Jo' par M. Joseph Caillaux", Le Figaro, 16 de marzo de 1914, p. 1.
} 
globo) en las semanas previas al estallido de la Gran Guerra. ${ }^{5}$

Horas después, los detalles sobre este episodio eran conocidos en Buenos Aires gracias a la virtual instantaneidad informativa que posibilitaba la inserción de la prensa porteña en la red global de distribución de las noticias. ${ }^{6}$ En los días posteriores, los entretelones del caso fueron seguidos y comentados con diversos grados de intensidad en todo el sistema de la prensa de Buenos Aires: los grandes matutinos, los vespertinos, los semanarios ilustrados, la prensa de izquierdas, la católica y, por supuesto, en las publicaciones de la colectividad francesa.

Y si bien los ecos de este affaire se hicieron más espaciados durante los meses de mayo y junio, sus ribetes folletinescos recobraron su espectacularidad a partir del 20 de julio de 1914 con el inicio del proceso contra Mme. Caillaux en la Cour d'assises de París,

\footnotetext{
${ }^{5}$ Jean-Jacques Becker, 1914: Comment les Français son entrés dans la guerre. Contribution à l'étude de l'opinion publique printemps-été 1914, París, Presses de la Fondation National de Sciences Politiques, 1977, pp. 131-136. Berenson, en el libro antes citado, menciona las repercusiones del caso en Alemania, Inglaterra y los Estados Unidos (p. 4), aunque no existen trabajos monográficos al respecto. Para el caso de América Latina, una simple ojeada a la prensa brasileña de la época, por ejemplo, muestra la significativa presencia del caso en las principales publicaciones periódicas del Brasil. Por su parte, el joven Mariátegui, en una crónica publicada en La Prensa de Lima el $1^{\circ}$ de agosto de 1914, comentó extensamente el caso. Véase José Carlos Mariátegui, "Cuenta el cable", en Antología (selección, introducción y notas de Martín Bergel), Buenos Aires, Siglo XXI, 2020, pp. 213215. Agradezco a Martín Bergel por esta referencia.

${ }^{6}$ A modo de ejemplo: "Francia. Desenlace de una campaña política. Drama en 'Le Fígaro'”, La Nación, 17 de marzo de 1914, p. 6. Sobre la incorporación de la prensa local al entramado informativo de las agencias de noticias véase Lila Caimari, "En el mundo barrio. Circulación de noticias y expansión informativa en los diarios porteños del siglo XIX”, Boletín del Instituto de Historia Argentina y Americana "Dr. Emilio Ravignani", $3^{\text {a }}$ Serie, $n^{\circ} 49,2018$, pp. 81-116 y "News from Around the World: The Newspapers of Buenos Aires in the Age of the Submarine Cable, 1866-1900", Hispanic American Historical Review, vol. 96, n 4, 2016, pp. 607-640.
}

cuyas características de funcionamiento le otorgaban al juicio una dimensión de espectáculo teatral, ampliamente explotado por la prensa parisina. ${ }^{7}$ Tan solo ocho días después, en coincidencia con el estallido de la Guerra Austro-serbia, el abogado Fernand Labori -que había participado en otros procesos resonantes como la defensa del anarquista Auguste Vaillant y de Lucie Dreyfus, esposa del célebre capitán francés- obtuvo la absolución de la acusada bajo la figura del crimen pasional; pues, apelando a la "debilidad emocional" de su defendida -considerada como una característica intrínseca de su condición de mujer- logró justificar el atentado contra Calmette como un acto "irracional". 8 Como reconocía un anónimo comentarista del diario La Razón a raíz de la finalización del juicio, el "drama de $L e F i$ garo" contaba con los elementos suficientes como para escribir un libro. ${ }^{9}$

A pesar de su brevedad, el affaire Caillaux constituye una coyuntura de gran densidad para analizar algunos de los rasgos constitutivos de la prensa de Buenos Aires y de la cultura mediática porteña de comienzos del siglo xx. Más allá de las condiciones materiales que posibilitaban el rápido acceso a la información europea, la amplia repercusión a nivel local que despertó este crimen se explica, en primer lugar, por la extendida francofilia de las élites políticas y culturales de Buenos Aires y por el lugar otorgado a la ciudad de París en el imaginario de los porteños. ${ }^{10}$ Fruto de

\footnotetext{
7 Una descripción del funcionamiento de la Cour d'assises puede verse en Berenson, The Trial of Madame Caillaux, pp. 5-6.

8 Jérôme Grévy, "Criminelle mais non déviante, madame Caillaux", en F. Chauvaud y G. Malandain, Impossibles victimes, impossibles coupables. Les femmes devant la justice (xix $x^{e}-x x^{e}$ siècles), Rennes, Presses universitaires de Rennes, 2009, pp. 229-237.

9 “El quinto: no matar", La Razón, 29 de julio de 1914, p. 1 .

${ }^{10}$ Véase Hebe Carmen Pelosi, Argentinos en Francia, franceses en Argentina. Una biografía colectiva, Buenos
} 
ese afrancesamiento, de una $\mathrm{u}$ otra forma, buena parte de lo que ocurría en la capital francesa era objeto de una atención particular en la prensa local.

No obstante, el affaire Caillaux permite abordar otras aristas de esa francofilia y constatar la pervivencia en un amplio sector de la prensa porteña $\mathrm{y}$, en especial, en los grandes matutinos, del modelo periodístico del quotidien d'information. ${ }^{11}$ En este sentido, Le Figaro (epicentro del drama) era un diario conocido y respetado en Buenos Aires por la excelencia de su confección y la calidad de su plantel de colaboradores, entre los que se contaba el escritor y periodista uruguayo Eugenio Garzón, responsable de la sección fija dedicada a América Latina. ${ }^{12}$ Esa influencia puede verse tanto en las semejanzas formales y de diseño presentes en diarios porteños como $L a$ Prensa o La Nación, como en las frecuentes referencias a Le Figaro por parte de figuras muy afrancesadas del periodismo local como era el caso del Emir Emin Arslán, entre otros. ${ }^{13}$ Ese modelo periodístico estaba asociado también a un determinado perfil de director que cultivaba los vínculos con el universo cultural y literario parisino, sin perder de vista la administración profesional de las facetas comer-

Aires, Ciudad Argentina, 1999, pp. 25-58; y Denis Rolland, La crise du modèle français. Marianne et l'Amérique latine. Culture, politique et identité, París, L'Harmattan, 2011 [2000].

${ }^{11}$ Para un análisis de este tipo de periódicos véase Dominique Kalifa, Marie-Ève Thérenty y Alain Vaillant, "Le quotidien", en D. Kalifa et. al., La civilisation du journal. Histoire culturelle et littéraire de la presse française au XIX siécle, París, Nouveau Monde éditions, 2011, pp. 269-294.

${ }^{12}$ Una historia de este matutino puede consultarse en Claire Blandin (dir.), Le Figaro. Histoire d'un journal, París, Nouveau Monde éditions, 2014.

${ }^{13}$ Arslán, que vivió en París entre 1893 y 1910, señala con frecuencia a Le Figaro en sus textos en la revista $L a$ Nota (1915-1921), que había creado en agosto de 1915. Véase, entre otros, "A modo de prefacio", La Nota, n ${ }^{\circ}$, 14 de agosto de 1915, pp. 1-2; y "La condición de los intelectuales en la Argentina", La Nota, n 19, 18 de diciembre de 1915, pp. 359-361. ciales y empresariales de un matutino de esa envergadura. ${ }^{14}$

Cabría señalar también que, dadas las características de este affaire, varios de los artículos publicados en los diarios porteños merodeaban la cuestión de los límites éticos y morales de la prensa moderna. ¿En qué medida era posible vulnerar la esfera de la vida privada y la intimidad de un hombre público en nombre de la primicia o de una campaña política? Ante este interrogante sobre los alcances de lo noticiable, el grueso de la prensa de Buenos Aires consideró que el director de Le Figaro había violado principios básicos de la deontología profesional al publicar unas cartas privadas obtenidas de manera espuria y que se trataba de un hecho repudiable aunque, por supuesto, ello no justificaba el violento accionar de la mujer de Caillaux.

Todos estos aspectos, que emergen a la luz del "crimen de Le Figaro", revelan que, a pesar del incipiente proceso de "norteamericanización" de la prensa porteña y la difusión del modelo sensacionalista del "nuevo periodismo" americano, cuya presencia en Buenos Aires se remonta a fines del siglo XIX y que en las primeras décadas del siglo $\mathrm{xx}$ tuvo una buena acogida en algunos vespertinos como Crítica, ${ }^{15}$ no debe perderse de vista que la prensa y el periodismo francés continuaban ejerciendo una gran influencia en sus colegas del Río de la Plata.

Ahora bien, más allá de la circulación de estos modelos periodísticos franceses en Buenos Aires, la francofilia que emerge asociada

${ }^{14}$ Recuérdese, a modo de ejemplo, que Marcel Proust había dedicado a Calmette su libro Du côté de chez Swann (Grasset, 1913) "como testimonio de un profundo y afectuoso reconocimiento". Asimismo, en el momento de ingresar a la redacción del diario el día del atentado, Calmette iba acompañado por el famoso novelista Paul Bourget.

${ }^{15}$ Sobre la influencia del modelo norteamericano véase Sylvia Saítta, Regueros de tinta. El diario Crítica en la década de 1920, Buenos Aires, Sudamericana, 1998. 
a las resonancias de este caso policial y judicial puede ser pensada también como un espacio de disputa en torno a diferentes representaciones sobre Francia. Por un lado, la imagen del Hexágono asociada a los ideales de la Ilustración y los principios de la Revolución de 1789 que, desde mediados del siglo XIX, fueron progresivamente fusionados con la latinidad como una ideología legitimadora de la expansión francesa en Sudamérica. Desde esa perspectiva, que puede apreciarse en las notas y artículos dedicados a los festejos del 14 de julio en la prensa de Buenos Aires, ${ }^{16}$ Francia era percibida como un paradigma literario y estético, identificado a una lengua y a un sistema de valores en cuyo centro se hallaba la ciudad de París, parada obligada en el grand tour de las élites argentinas por el Viejo Continente. ${ }^{17}$

Pero, por otro lado, el affaire Caillaux dinamizó otras representaciones sobre Francia vinculadas a una mirada conservadora y mucho más crítica de la Tercera República. En este sentido, a raíz de la decisión del jurado de absolver a la acusada, El Diario publicó un extenso comentario que denunciaba la corrupción de la política francesa: "Divorcios, amantes, chantages, delaciones; un desfile siniestro de todas las pobrezas del corazón, un conjunto en el cual, sobre los cráneos alucina-

\footnotetext{
${ }^{16}$ Véanse, entre otras, El Diario. 14 julliet 1789-1914, suplemento especial, 14 de julio de 1914; "La fiesta de Francia", El Nacional, 14 de julio de 1914, p. 5; "El 14 de julio", La Argentina, 14 de julio de 1914, p. 4; y "Allons enfants de la Patrie", Le Courrier de la Plata, 14 de julio de 1914, p. 3.

${ }^{17}$ Sobre la importancia del viaje a París en las élites porteñas véase David Viñas, "La mirada de Europa: del viaje colonial al viaje estético", en Literatura argentina y política I. De los jacobinos porteños a la bohemia anarquista, Buenos Aires, Santiago Arcos, 2005 [1964], pp. 11-67, y Beatriz Colombi, "Camino a la meca: escritores hispanoamericanos en París (1900-1920)", en C. Altamirano (dir.) y J. Myers (ed.), Historia de los intelectuales en América Latina I. La ciudad letrada, de la conquista al modernismo, Buenos Aires, Katz, 2008, pp. 544-566.
}

dos de los políticos bailan un can-can desenfrenado los zapatos de las cocotas". A partir del tópico finisecular de la décadence, este vespertino situaba al affaire Caillaux como un acontecimiento ejemplificador de la degradación de la clase política que dejaba al descubierto "la miseria moral de nuestra época" y "la espantosa desorientación en la que vivimos". Como corolario, El Diario lanzaba una advertencia sobre la pérdida del rumbo de una nación fuertemente influyente para la Argentina: "No es Francia el baile que se ofrece en los cabarets al extranjero que después de todo solo eso quiere ver. Es el gran pueblo de la libertad, la justicia y el ahorro, hoy víctima, como todos, de la falta de norte moral". ${ }^{18}$

Esa representación de una Francia extraviada como consecuencia de los males de la modernidad fue reiterada con mayor virulencia en las páginas del diario católico El Pue$b l o$, en un inflamado editorial que retomaba la imagen de las dos Francias, representadas de un modo simbólico en las ciudades de Lourdes y París:

Ahí están de frente, en campos bien deslindados, las dos Francias de la hora actual [...] Ahí está la Francia de Jesucristo en Lourdes y la Francia atea de la corte de asises. En Lourdes está la Francia de San Dionisio y sus compañeros mártires, la de San Martín y San Hilario; la Francia de Martel, de Carlo Magno y de San Luis; la de San Vicente de Paul y de San Juan de la Salle; la Francia de misioneros incansables é intrépidos [...] En el otro campo aparece, la Francia del terror y de la guillotina, de la masonería insidiosa y siniestra, de la Comuna incendiaria, de Venus endiosada, del divorcio rayano en el amor libre y de la persecución y de la intolerancia sectarias.

\footnotetext{
18 "Derecho a matar", El Diario, 29 de julio de 1914, p. 4.
} 
En una palabra, en la corte de asises está la mujer atea y divorciada que asesina, y defendiéndola aparecen los hombres políticos que desgraciadamente tienen en sus manos las riendas del gobierno [...] En París, en el tribunal que juzga á la mujer divorciada, asesina de Calmette, está esa parte de Francia entronizada en el poder que aborrece á sus hermanos, los franceses católicos [...] la Francia de Gambetta, suicida; de Waldeck Rousseau, engañador; de Combes, que gobernó pagando el espionaje y la delación; de Zola, el asqueroso de mala fe [...] esa parte de Francia que Maurice Barrés pintó con pluma magistral en los artículos titulados "Dans la cloaque". ${ }^{19}$

La figura de Mme. Caillaux opera aquí como una sinécdoque de Francia pues todos los males que ella encarna son el resultado de un proceso de secularización impulsado por el laicismo republicano, que habían torcido el destino de la nación católica y monárquica.

En segundo lugar, aunque vinculado a esto último, por el hecho de que su protagonista fuera una mujer casada en segundas nupcias luego de que Caillaux obtuviera su divorcio, ${ }^{20}$ y por la manera en que la cultura jurídica francesa de la época resolvió el caso, este escándalo se transforma también en un momento propicio para observar algunas de las representaciones sobre la mujer y su rol en la sociedad contemporánea que circulaban en la prensa porteña. Mientras que algunos periódicos más liberales miraban con simpatía a la figura de Henriette, en los periódicos vinculados al catolicismo las críticas hacia la protagonista del caso fueron demoledoras. Con

19 "Las dos Francias", El Pueblo, 26 de julio de 1914, p. 2.

${ }^{20}$ Sobre este aspecto, véase Edward Berenson, "The Politics of Divorce in France of the Belle Époque: The Case of Joseph and Henriette Caillaux", The American Historical Review, vol. 93, n 1, 1988, pp. 31-55. motivo de la absolución de la acusada, El Pueblo publicó otro duro editorial titulado "La madama absuelta" en el cual volvía a arremeter contra Mme. Caillaux considerándola como "el producto genuino de la moral sin Dios implantada en las escuelas y practicada en todos los órdenes de la vida social contemporánea". ${ }^{21} \mathrm{Y}$ al igual que en su comentario anterior, el diario católico no ahorraba vituperios contra la acusada: "mujer adúltera, perturbadora del hogar ajeno en mira de un provecho propio, divorciante, divorciada, vuelta á casar, lasciva como una mona" eran solo algunos de los insultos proferidos desde sus páginas. No obstante, en ocasiones, esa mirada misógina y despectiva se hizo extensiva al jurado responsable de su absolución mediante la atribución de rasgos de debilidad típicos de la condición femenina. En este sentido, El Diario en el artículo antes citado cargaba contra los errores de ese "jurado sentimental, con alma de lector de folletín", que había mostrado unas "sensiblerías de costurera ante un crimen que exigía serenidad y energía de hombre". ${ }^{22}$

En tercero y último lugar, el análisis de las repercusiones locales del affaire Caillaux permite mostrar el eclecticismo temático y la volatilidad de los intereses del público porteño y de esa "cultura de la noticia", alimentada por el flujo permanente de la información internacional que provenía de las agencias de noticias europeas y norteamericanas. De hecho, si se compara la importancia otorgada al "drama de Le Figaro" pero también a los entretelones del "crimen de la calle Gallo" (otro resonante caso policial aunque de un alcance más local), ${ }^{23}$ respecto de las repercusiones del

21 "La madama absuelta", El Pueblo, 30 de julio de 1914, p. 2.

22 "Derecho a matar", El Diario, 29 de julio de 1914, p. 4.

${ }^{23}$ El 19 de julio de 1914, Frank Carlos Livingston, subcontador del Banco de la Nación Argentina, fue brutal- 
atentado de Sarajevo y la crisis diplomática de julio de 1914, es evidente que los primeros acapararon de un modo más intenso la atención de la prensa y el público porteño. Ese relativo desinterés frente a la crisis europea se mantuvo hasta el estallido de la Guerra Austro-serbia, cuyos inicios coincidieron con la finalización del juicio contra Mme. Caillaux, mostrando una dinámica similar a lo ocurrido en Francia y en otras partes de Europa. ${ }^{24}$

Cuando la prensa porteña descubre la gravedad del problema, la "crisis" se había transformado ya en una "guerra europea". A partir

mente apuñalado en su casa en la calle Gallo 1680. La investigación policial descubrió que la mujer del fallecido, Carmén Guillot, había planeado con ayuda de su mucama y confidente, Catalina González de Corello, un complot para asesinar a su marido que, al parecer, la engañaba con varias amantes. Para concretarlo, recurrió a los servicios de Salvatore Viterale, un vendedor de pescado y amigo de la mucama, que fue el encargado de reclutar a sus cómplices -Raffaele Prestamo, Francesco Salvatto y Giacommo Battista Lauro-, todos inmigrantes italianos y compañeros de trabajo de Viterale. Por el hecho, Carmen Guillot fue condenada a reclusión por tiempo indeterminado mientras que Lauro y Salvato fueron condenados a pena de muerte, la última ejecutada en la Argentina, el 22 de junio de 1916.

${ }^{24}$ Las crónicas de los corresponsales instalados en París y en otras ciudades del Viejo Continente revelan ciertas semejanzas con las reacciones de la prensa y la opinión pública europea. "Había que ver, ayer, el aspecto de los bulevares de 10 á 12 de la noche y aun hasta las 2 de la madrugada", comentaba Pedro S. Lamas, director de la Revue Sudamericaine y corresponsal del diario La Razón en París, en una crónica dedicada a la absolución de la acusada. "Las ediciones de los diarios eran arrebatadas, se agotaban y se renovaban sin cesar, pregonando la absolución de la asesina con asombro, con indignación de parte de cierto público, especialmente del femenino, con satisfacción y aplauso de parte de muchos". Pedro S. Lamas, "Epílogo del asunto Caillaux - Calmette. El apasionamiento público ante el veredicto", La Razón, 22 de agosto de 1914, p. 4. No obstante, ese tipo de reacciones no fueron exclusivas de la capital francesa. Desde Bruselas, el corresponsal de La Nación, Roberto Payró, afirmaba en la crónica que sirve de acápite a este artículo: "El proceso de Mme. Caillaux absorbe de tal modo la atención pública que ni siquiera se habla, sino muy incidentalmente, en estos momentos, del terrible ultimátum de Austria a Serbia, aunque vaya en ello envuelta la paz europea". Roberto J. Payró, "Cartas informativas", p. 4. de entonces, los hechos se sucedieron con gran velocidad: el asesinato de Jean Jaurès; la declaración de guerra entre las grandes potencias y la orden de movilización general; la invasión alemana de Bélgica; el ingreso de Inglaterra en el conflicto y la declaración de la neutralidad argentina, ocurrida el 4 de agosto de 1914. Súbitamente, la Gran Guerra devino un asunto excluyente para la prensa y la opinión pública de Buenos Aires, desplazando todos los otros temas de interés y dando inicio a una primera fase en la cobertura periodística del conflicto que se prolongó hasta finales de ese año. ${ }^{25}$

Paradójicamente, el mismo día que el Estado argentino declaró su neutralidad ante el conflicto bélico fue detenido Giacommo Battista Lauro, el último de los prófugos del "crimen de la calle Gallo", cuya captura pasó casi desapercibida, sepultada bajo el cúmulo de informaciones sobre la guerra:

Días atrás, la noticia habría causado sensa-
ción. No habría llegado por otra parte, de
improviso, pues el público, que seguía an-
siosamente la campaña de los pesquisas,
hubiera sabido que la detención de Lauro
era inminente. Las gentes se dedicarían a
comentar el suceso y la policía mostraríase
ufana de su éxito ruidoso. Hoy la informa-
ción carece de interés y el público se ha
enterado de ella con indiferencia. Absor-
bida la atención general con los sucesos
europeos, ¿qué puede importarle la captura
de un asesino calabrés? Lauro no ha tenido
suerte [...] no tendrá siquiera como sus
cómplices la satisfacción de una notorie-
dad escandalosa, pues la formidable catás-

${ }^{25}$ Sobre la fascinación provocada por el estallido de la guerra véase Emiliano Gastón Sánchez, "Pasión de multitudes: la prensa y la opinión pública de Buenos Aires frente al estallido de la Gran Guerra", Anuario IEHS, vol. $33, \mathrm{n}^{\circ} 1,2018$, pp. 177-204. 
trofe europea no permite al público enterarse por su suerte. ${ }^{26}$

La sorna del comentarista del diario La Mañana sobre la "mala suerte" del último prófugo por el asesinato de Livingston, al coincidir su captura con la masificación de la guerra en Europa, muestra la volatilidad de las expectativas y los intereses de los lectores de Buenos Aires y la rapidez con la que los temas pasaban de ser el comentario del momento a una noticia intrascendente. Una dinámica noticiosa a la que tampoco pudo escapar el estridente affaire Caillaux.

\section{Bibliografía citada}

Becker, Jean-Jacques, 1914: Comment les français son entrés dans la guerre. Contribution à l'étude de l'opinion publique printemps-été 1914, París, Presses de la Fondation National de Sciences Politiques, 1977.

Berenson, Edward, The Trial of Madame Caillaux, California, University of California Press, 1992.

"The Politics of Divorce in France of the Belle Époque: The Case of Joseph and Henriette Caillaux", The American Historical Review, vol. 93, $\mathrm{n}^{\circ}$ 1, 1988, pp. 31-55.

Blandin, Claire (dir.), La Figaro. Histoire d'un journal, París, Nouveau Monde éditions, 2014.

Caimari, Lila, "En el mundo-barrio. Circulación de noticias y expansión informativa en los diarios porteños del siglo XIX", Boletín del Instituto de Historia Argentina

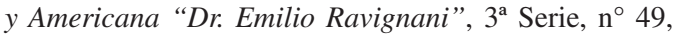
2018, pp. 81-116.

26 "El pobre Lauro", La Mañana, 4 de agosto de 1914, p. 1 .
_ of Buenos Aires in the Age of the Submarine Cable, 1866-1900", Hispanic American Historical Review, vol. 96, $\mathrm{n}^{\circ} 4$, Durham, Duke University Press, noviembre de 2016, pp. 607-640.

Colombi, Beatriz, "Camino a la meca: escritores hispanoamericanos en París (1900-1920)", en C. Altamirano (dir.) y J. Myers (ed.), Historia de los intelectuales en América Latina I: La ciudad letrada, de la conquista al modernismo, Buenos Aires, Katz, 2008, pp. 544-566.

Grévy, Jérôme, "Criminelle mais non déviante, madame Caillaux", en F. Chauvaud y G. Malandain, Impossibles victimes, impossibles coupables. Les femmes devant la justice (XIX $-X X^{e}$ siècles), Rennes, Presses universitaires de Rennes, 2009, pp. 229-237.

Kalifa, Dominique, Marie-Ève Thérenty y Alain Vaillant, "Le quotidien", en D. Kalifa et al., La civilisation du journal. Histoire culturelle et littéraire de la presse française ai XIX ${ }^{e}$ siécle, París, Nouveau Monde éditions, 2011, pp. 269-294.

Le Naour, Jean-Yves, Meurtre au Figaro. L'affaire Caillaux, París, Larousse, 2007.

Mariátegui, José Carlos, Antología (selección, introducción y notas de Martín Bergel), Buenos Aires, Siglo XXI editores, 2020 .

Pelosi, Hebe Carmen, Argentinos en Francia, franceses en Argentina. Una biografía colectiva, Buenos Aires, Ciudad Argentina, 1999.

Rolland, Denis, La crise du modèle français. Marianne et l'Amérique latine. Culture, politique et identité, París, L'Harmattan, 2011 [2000].

Saítta, Sylvia, Regueros de tinta. El diario Crítica en la década de 1920, Buenos Aires, Sudamericana, 1998.

Sánchez, Emiliano Gastón, "Pasión de multitudes: la prensa y la opinión pública de Buenos Aires frente al estallido de la Gran Guerra", Anuario IEHS, vol. 33, n 1 , 2018, pp. 177-204.

Viñas, David, "La mirada de Europa: del viaje colonial al viaje estético", en Literatura argentina y política I. De los jacobinos porteños a la bohemia anarquista, Buenos Aires, Santiago Arcos, 2005 [1964], pp. 11-67. 


\section{Resumen/Abstract}

Notas sobre las repercusiones del affaire Caillaux en la prensa de Buenos Aires

El objetivo de este artículo es analizar las repercusiones en la prensa de Buenos Aires del affaire Caillaux, un escándalo político y mediático que mantuvo en vilo a Francia en las semanas previas al estallido de la Gran Guerra. A pesar de su brevedad, la coyuntura abierta por este caso permite analizar diversos rasgos de la prensa de Buenos Aires y de la cultura mediática porteña de comienzos del siglo xx. Entre las cuales, cabría señalar: la circulación de los modelos periodísticos franceses en Buenos Aires y el eclecticismo temático que caracterizó a los intereses del público porteño como consumidor de las noticias internacionales.

Palabras claves: Affaire Caillaux - Prensa periódica - Buenos Aires - Gran Guerra

DOI: https://doi.org/10.48160/18520499prismas25.1221
Notes on repercussions of the Caillaux affair in the Buenos Aires periodical press

The objective of this article is to analyze the repercussions in the Buenos Aires press of the Caillaux affair, a political and media scandal that kept France in suspense in the previous weeks at the outbreak of the Great War. Despite its brevity, the conjuncture opened by this case allows us to analyze some of the features of the Buenos Aires press and the Buenos Aires media culture of the early twentieth century. Among which, it should be noted: the circulation of French journalistic models in Buenos Aires and the thematic eclecticism that characterized the interests of the Buenos Aires public as consumers of international news.

Keywords: Affaire Caillaux - Periodical Press Buenos Aires - Great War 